19 Revue d'histoire du XIXe siècle

Société d'histoire de la révolution de 1848 et des

révolutions du XIXe siècle

22 | 2001

Autour de Décembre 1851

Marcel Vigreux (1933-2001)

Pierre Lévêque

URL : http://journals.openedition.org/rh19/284

DOI : $10.4000 /$ rh19.284

ISSN : $1777-5329$

Éditeur

La Société de 1848

Édition imprimée

Date de publication : 1 juin 2001

ISSN : 1265-1354

Référence électronique

Pierre Lévêque, "Marcel Vigreux (1933-2001) », Revue d'histoire du XIXe siècle [En ligne], 22 | 2001, mis en ligne le 04 septembre 2008, consulté le 15 septembre 2020. URL : http://journals.openedition.org/ rh19/284

Ce document a été généré automatiquement le 15 septembre 2020

Tous droits réservés 


\title{
Marcel Vigreux (1933-2001)
}

\author{
Pierre Lévêque
}

1 Le 17 juillet 2001, notre collègue Marcel Vigreux nous a quittés après une longue et douloureuse maladie contre laquelle il a lutté avec un très grand courage.

Morvandiau de Paris (où son père était fonctionnaire de police), il a manifesté toute sa vie un attachement profond à son pays d'origine. D'abord instituteur, puis professeur agrégé au lycée d'Autun après ses études supérieures à la Sorbonne, il entreprit sous la direction d'André Armengaud la préparation d'une thèse de troisième cycle sur La Société d'agriculture d'Autun au XIXe siècle. Il la soutint en 1970, alors qu'il était depuis trois ans déjà assistant d'histoire contemporaine à l'Université de Dijon, où il allait poursuivre sa carrière jusqu'à sa retraite.

Enseignant de grande valeur, ses étudiants ont gardé le souvenir des qualités - précision, clarté, autorité - qui caractérisaient ses commentaires de documents et ses cours magistraux. Il excellait en particulier à faire revivre et comprendre l'histoire des campagnes françaises, à laquelle il venait de consacrer un recueil de documents (Les crises du monde rural, 1970).

Pendant la décennie suivante, avec une minutie passionnée, il a accumulé les matériaux très divers (des archives nationales, départementales, communales et privées à l'enquête orale) qui lui ont permis d'édifier, sous la direction de Louis Girard, puis de Maurice Agulhon, sa grande thèse centrée sur l'étude des rapports entre Paysans et notables du Morvan au XIXe siècle. Ayant pris pour point de départ les années 1840, il a bien entendu rencontré et analysé en profondeur la longue crise de 1848-1851: membre actif de la Société d'histoire de la révolution de 1848 , il a présenté de façon lumineuse cette étape décisive de la politisation des ruraux dans une région moins isolée qu'on ne l'a cru, avant de consacrer des chapitres tout aussi éclairants à la reprise en main de la paysannerie par les notables, les pouvoirs publics et l'Église au temps de l'Empire et de l'Ordre moral, puis à son émancipation progressive sous l'influence de la petite et moyenne bourgeoisie républicaine, des années 1870 à la veille de la Première Guerre mondiale. Grâce à lui, le Morvan devenait une des régions de France les mieux connues dans son évolution économique, sociale et politique contemporaine. Docteur ès lettres et sciences humaines en juin 1985, Marcel Vigreux obtint quelque temps après un poste de professeur à l'Université de Bourgogne. 
Dans le même temps, il a joué un rôle capital pour le développement de la recherche et de la mémoire historique dans sa région : direction de travaux d'étudiants, publication d'articles de fond et édition de documents dans le Bulletin de l'Académie du Morvan dont il avait pris la responsabilité dès 1974 . Témoin dans son enfance de l'action des maquisards, il créa en 1976, avec le doyen Jean-René Suratteau, le Centre d'études et de recherches sur l'occupation et la résistance en Morvan (CERORM), chargé en particulier de stimuler et de faciliter les travaux, de recueillir et de publier des témoignages, d'organiser des conférences avec l'appui du Parc naturel régional et de l'Université de Bourgogne. Une remarquable exposition organisée en 1979 à la Maison du Parc à SaintBrisson allait se transformer en un Musée permanent de la Résistance en Morvan, inauguré le 26 juin 1983 par le président François Mitterrand, en présence de nombreux anciens combattants de la clandestinité.

C'est aussi à Marcel Vigreux, seul ou aidé par d'anciens étudiants, que l'on doit la publication de précieux recueils de témoignages et d'études sur la période 1940-1944. Citons simplement La mémoire de Dun-les-Places (1990), Les villages martyrs de Bourgogne (1994), Le Morvan pendant la Seconde Guerre mondiale (1996).

Depuis 1993, Marcel Vigreux vivait une retraite très active et assumait de multiples responsabilités : président de l'Académie du Morvan, premier vice-président du Parc, puis président de son Conseil scientifique, et même, en fonction de l'intérêt qu'il avait toujours porté à la culture populaire, président du jury international des Folkloriades de Dijon. Depuis 1966, il exerçait les fonctions de maire de Ménessaire, cette commune côte-d'orienne du haut Morvan enclavée entre Nièvre et Saône-et-Loire, où avaient vécu ses ancêtres. De la maison qu'il s'y était fait construire en haut du village, il pouvait contempler un de ces paysages morvandiaux dont il savait si bien faire ressortir l'originalité et le charme, héritage d'une longue évolution historique.

À la fois universitaire et homme d'un terroir, associant harmonieusement enseignement, recherche scientifique, vulgarisation de haut niveau, activités culturelles et engagement civique, Marcel Vigreux vivra dans le souvenir fidèle de tous ceux qui l'ont connu.

Pierre Lévêque est professeur honoraire à l'Université de Bourgogne

\section{Bibliographie de Marcel Vigreux (concernant l'histoire du XIXe siècle)}

Auteuil pendant la Révolution française, mémoire sous la direction de M. Merlier, École normale d'instituteurs d'Auteuil, 1953, 96 fo (inédit)

Monographie de Ménessaire, Côte-d'Or, mémoire sous la direction de M. Merlier, École normale d'instituteurs d'Auteuil, 1954, 252 fo (inédit)

Evolution de l'économie et de la société rurales dans une commune du Morvan (Ménessaire), au XIXe siècle, Diplôme d'études supérieures sous la direction de Louis Girard, Faculté des lettres de Paris, 1957, 183 fo (inédit)

Les crises du monde rural, Dossier sciences humaines $n^{\circ} 23$, Paris, Librairie Armand Colin, $1970,96 \mathrm{p}$.

"La vie dans la forêt", dans Courrier du Parc naturel régional du Morvan, n 4, hiver 1971, pp. 13-15.

"La Société d'Agriculture d'Autun (1833-1914)", dans Bulletin de l'Académie du Morvan, $\mathrm{n}^{\circ}$ 1, 1974, pp. 3-20. 
"Coup d'œil sur l'histoire du Morvan", dans, Le Morvan et son PNR.- Annales des pays nivernais, Camosine, n 9, 1975, pp. 20-21.

"La chaumière morvandelle", dans Le journal du Centre, 2 avril 1977 et Le Courrier de Saône-et-Loire, 25 janvier 1977 et 26-27 février 1977.

"Saisie d'un collecteur de tailles à Saint-Brisson [N.] en 1768", dans Bulletin de l'Académie du Morvan, $\mathrm{n}^{\circ}$ 5, 1977, pp. 27-28 (en collaboration avec Joseph ROBLIN).

"Des paysans républicains à la fin du Second Empire : les élections de 1869 dans le Morvan nivernais", dans Revue d'histoire moderne et contemporaine, tome 25, 1978, pp. 443-469.

"Les démocrates socialistes dans le haut Morvan (1849-1852)", dans Congrès de l'Association Bourguignonne des Sociétés Savantes, Nevers, 1978, publiés en 1979 par la Société académique du Nivernais, pp. 129-138.

"La Maison du Parc à Saint-Brisson : son histoire", dans Courrier du Parc naturel régional du Morvan, $\mathrm{n}^{\circ} 19$ 1979, pp. 4-9 (en collaboration avec Joseph ROBLIN).

"La chaumière morvandelle, témoin d'une civilisation", dans Courrier du Parc naturel régional du Morvan, $\mathrm{n}^{\circ}$ 19, 1979, pp. 10-14.

Le centre de lecture du paysage en Morvan.- Courrier du Parc naturel régional du Morvan, $\mathrm{n}^{\circ} 21,1979$ [Coordination du numéro, de sa plume : préambule, bibliographie, lexique]

Les Patois du Morvan.- Courrier du Parc naturel régional du Morvan, $\mathrm{n}^{\circ} 23,1980$ [Coordination du numéro, de sa plume: "Les patois du Morvan", p. 8 ; "Mesures agraires et forestières", pp. 24-27 ; "Bibliographie", pp. 34-35; "Sérieux et facétie des Morvandiaux" (en collaboration avec Claude RÉGNIER), pp. 36-37].

Les Forêts morvandelles.- Courrier du Parc naturel régional du Morvan, $\mathrm{n}^{\circ} 25,1981$ [Coordination du numéro, de sa plume: "Place et rôle de la forêt traditionnelle", pp. $2-7$; "Une révolution économique pour le Morvan : le flottage du bois", pp. 8-14].

"L'école en Morvan au XIXe siècle", dans Bulletin de l'Académie du Morvan, n 13, 1981, pp. 28-36 [trois textes présentés avec Julien DACHÉ].

La Galvache et les galvachers: une migration morvandelle, Autun, Imprimerie Pelux, 1982, $38 \mathrm{p}$.

"La forêt morvandelle : passé, présent", dans Les Annales des pays nivernais, Camosine, $\mathrm{n}^{\circ}$ 33, 1982, pp. 21-23.

"Paris possédait-il le Morvan au XIXe siècle ?", dans Actes du 52e Congrès de l'Association bourguignonne des sociétés savantes, Avallon, Vézelay, Château-Chinon, (1981), 1982, pp. 47-57.

"La préservation des fortunes foncières en Morvan au XIXe siècle", dans Bulletin du Centre d'histoire économique et sociale de la région lyonnaise, 1982, $\mathrm{n}^{\circ}$ 3, pp. 43-53.

"Une expérience agricole originale dans l'Autunois au XIXe siècle : la ferme-école de Tavernay (1840-1850)", dans 53e Congrès de l'Association bourguignonne des sociétés savantes, Le Creusot (juin 1982), 1983, pp. 183-193.

La chasse en Morvan.- Courrier du Parc naturel régional du Morvan, $\mathrm{n}^{\circ} 32,1985$ [Coordination du numéro, de sa plume : "La chasse, phénomène de société", pp. 2-3 ; "De la chasse morvandelle à l'événement national : l'affaire Montcharmont (1850)", pp. 30-35.

Paysans et notables du Morvan au XIXe siècle jusqu'en 1914, Château-Chinon, Académie du Morvan, 1987, (Préface de Maurice Agulhon), 755 p. [réédition, 1998] [édition de Paysans et notables du Morvan au XIXe siècle (jusqu'en 1914), Thèse d'État sous la direction de Maurice Agulhon, Université Paris I, 1985, 4 volumes, $1042 \mathrm{f}^{\circ}+$ annexes), Prix Araxie Torossian, 1986, Académie des sciences morales et politiques et Prix littéraire du 
Morvan, 1988].

"L'industrie des nourrices morvandelles et des enfants assistés au XIXe siècle", dans Bulletin de l'Académie du Morvan, $\mathrm{n}^{\circ}$ 25, 1987, pp. 3-43.

"Protestation paysanne et contestation politique : l'exemple du Morvan sous la Seconde République", dans La protestation rurale dans les campagnes françaises. Questions à l'Histoire (XVIIe-XXe Siècle), Colloque de l'Association des ruralistes français, Arc-et-Senans, 1987, 3 p.

"Paysans et notables du Morvan au XIXe siècle", dans, Bulletin de l'Académie du Morvan, $\mathrm{n}^{\circ} 25,1987$, pp. 3-43.

"L'industrie des nourrices morvandelles et des enfants assistés au XIXe siècle", dans Annales du Midi. $\mathrm{n}^{\circ}$ 25, 1987, pp. 3-43.

"Comportements révolutionnaires en Morvan central au milieu du XIXe siècle: structures foncières, sociales et mentales, souvenir de l'Ancien régime et de la Révolution", dans Jean BART [dir.], Le Morvan révolutionnaire. Recherches sur les origines des traditions politiques en Morvan (XVIIIe-XIXe siècles), Paris, Société des études robespierristes/Comité départemental pour l'histoire de la Révolution française en Côte-d'Or, 1988, pp. 87-103. [cet ouvrage est un tiré à part de l'intégralité des articles du $\mathrm{n}^{\circ}$ 4-1988 des Annales historiques de la Révolution française].

"Le braconnier et le gendarme: l'affaire Montcharmont", dans L'Histoire, $\mathrm{n}^{\circ} 114$, septembre 1988, pp. 56-61.

"Nourrices et enfants assistés au XIXe siècle", dans Les Annales des pays nivernais, Camosine $\mathrm{n}^{\circ}$ 54, 1988, pp. 11-17.

La Société d'agriculture d'Autun (1833-1914), Dijon, EUD, 1989, 242 p. [édition de la Thèse de troisième cycle, 1970, 3 volumes, $416 \mathrm{f}^{\circ}$ ].

"Ethnographie" (en co-direction avec Jean DROUILLET avec la collaboration de Robert CHAPELIER, Capucine CROSNIER, François PORTET et Marcel POULET) dans NivernaisMorvan, Paris, Bonneton, 1989. [sous sa plume: "Clairières et vieilles techniques agricoles", "Métiers et usages du seigle", "Le flottage du bois", pp. 122-139, "La galvache et les galvachers", "Nourrices et enfants assistés", pp. 148-161, "Mentalités forestières du Haut-Nivernais", pp. 313-316 ; à noter également qu'il a participé à la partie "Art et histoire" dirigée par Jean-Pierre HARRIS].

"Comportements révolutionnaires en Morvan central au milieu du XIXe siècle", dans Du Nivernais à la Nièvre, études révolutionnaires, tome V, 1989.

"L'opposition des journaliers agricoles au maximum des salaires (1794)", dans L'Almanach du Morvan, n 1989, pp. 42-43.

"Un bail à métairie dans le département de la Nièvre en 1792", dans L'Almanach du Morvan, $\mathrm{n}^{\circ} 1989$ pp. 55-56

"Ethnographie", dans Yonne, Paris, Bonneton, [sous sa plume: "les "Petits Paris"", "Nourrices et enfants assistés", pp. 120-121].

"Troubles au village au XIXe siècle", dans Le Journal du Centre, n ${ }^{\circ} 35$, novembredécembre 1995.

"Une migration typiquement morvandelle: la Galvache", dans À la découverte de la Galvache et des Galvachers, 1995, pp. 2-3.

"L'industrie des nourrices morvandelles au XIXe siècle", dans Les nourrices, Dun-lesPlaces, Association historique de Dun-les-Places/PNRM, 1996, pp. 50-54. [1ère édition 1994].

"Préface" à Alain VIEILLARD-PASQUELIN, Au temps des galvachers, Précy-sous-Thil, Éditions de l'Armançon, 1997, 192 p. 
"Les Amis de la maison Vauban", dans Pays de Bourgogne, n 182, décembre 1998, p. 31 [en collaboration avec C. LEBOSSÉ].

"La poussée de la gauche "démoc-soc" dans la Nièvre : du succès de 1849 aux sociétés secrètes et aux répressions préventives", dans Coup d'État du 2 décembre 1851. Les insurgés de Clamecy et de la Nièvre. Actes du colloque de Clamecy, 24 mai 1997, Clamecy, Comité scientifique et artistique de Clamecy, s.d. [1998 ?], pp. 49-67.

"La Nièvre sous la Monarchie censitaire (1815-1848) et la Seconde République : la vie politique", dans André LEGUAI et Jean-Bernard CHARRIER [dir.], Histoire du Nivernais, Dijon, EUD, 1999, pp. 305-323.

"Le temps des notables (1852-vers 1880)", dans André LEGUAI et Jean-Bernard CHARRIER [dir.], Histoire du Nivernais, Dijon, EUD, 1999, pp. 336-343.

"Deux fermes écoles en Autunois et en Nivernais au milieu du XIXe siècle : Tavernay et Poussery", dans Michel BOULET [dir.], Les enjeux de la formation des acteurs de l'agriculture, 1760-1945, Actes du Colloque Enesad, 19-21janvier 1999, Dijon, Éditions Educagri, 2000, pp. 331-337.

"Le Morvan : une forte identité", dans Nièvre, vert pays des eaux vives, Nevers, Camosine, 2000, pp. 324-373.

"Préface" à Anne-Marie LAFAY, Autun à la fin du XIXe siècle, Château-Chinon, Académie du Morvan, 2000, 250 p.

"L'écomusée du Morvan et ses maisons à thèmes", dans Vents du Morvan magazine, $\mathrm{n}^{\circ} 4$, 2000, p. 2-8 [en collaboration avec Philippe HOELTZEL et Philippe GUILBERT].

"Les racines du socialisme nivernais: la place majeure de la Seconde République (1848-1851)", dans colloque Cent ans de socialisme dans la Nièvre, Recherches socialistes, 2001, [à paraître].

Muséographie :

Conception et réalisation de l'Écomusée du Morvan et de ses Maisons à thèmes, en particulier la Maison du seigle à Ménessaire.

Filmographie (conseiller historique pour les films suivants) :

La clairière... paysans et paysages, France 3 Bourgogne, 1997.

Le Morvan, pays de France. Faune et terroir, France 3 Bourgogne, 1997.

Les nourrices morvandelles, France 3 Bourgogne, 1998.

La Maison du seigle à Ménessaire, PNRM, 1999.

L'affaire Montcharmont [film en cours de réalisation].

\section{INDEX}

Mots-clés : Hommage 